\begin{tabular}{|c|l|}
\hline Title & The horospherical geometry of surfaces in Hyperbolic 4 space \\
\hline Author(s) & Izumiya, S.; Pei, D.; Romero-Fuster, M. C \\
\hline Citation & Hokkaido University Preprint Series in Mathematics, 573, 1-17 \\
\hline Issue Date & 2002-12 \\
\hline DOI & 10.14943/83718 \\
\hline Doc URL & http://hdl.handle.net/2115/69322 \\
\hline Type & bulletin (article) \\
\hline File Information & pre573.pdf \\
\hline
\end{tabular}

Instructions for use 
The horospherical geometry of surfaces in Hyperbolic 4-space

S. Izumiya, D. Pei and M.C. Romero-Fuster

Series \#573. December 2002 


\section{HOKKAIDO UNIVERSITY PREPRINT SERIES IN MATHEMATICS}

\#547 Y. Nakano, Minimizing coherent risk measures of shortfall in discrete-time models with cone constraints, 22 pages. 2002.

\#548 K. tachizawa, A generalization of the Lieb-Thirring inequalities in low dimensions, 13 pages. 2002.

\#549 T. Nakazi, Absolute values and real parts for functions in the Smirnov class, 8 pages. 2002.

\#550 T. Nakazi and T. Watanabe, Properties of a Rubin's orthogonal function which is a linear combination of two inner functions, 9 pages. 2002.

\#551 T. Ohtsuka, A level set method for spiral crystal growth, 24 pages. 2002.

\#552 M.-H. Giga and Y. Giga, Minimal vertical singular diffusion preventing overturning for the Burgers equation, 18 pages. 2002.

\#553 Y. Giga and P. Rybka, Berg's effect, 12 pages. 2002.

\#554 Y. Tonegawa, Domain dependent monotonicity formula for a singular perturbation problem, 14 pages. 2002.

\#555 S. Izumiya and N. Takeuchi, New special curves and developable surfaces, 10 pages. 2002.

\#556 S. Izumiya, K. Saji and N. Takeuchi, Singularities of line congruences, 17 pages. 2002.

\#557 S. Izumiya, D. Pei and M. C. Romero Fuster, The lightcone Gauss map of a spacelike surface in Minkowski 4-space, 21 pages. 2002.

\#558 S. Izumiya, D. Pei and M. C. Romero Fuster, Umbilicity of spacelike submanifolds of Minkowski space, 14 pages. 2002.

\#559 S. Izumiya, D. Pei and M. Takahashi, Curves and surfaces in Hyperbolic space, 16 pages. 2002.

\#560 J. Kato, On the uniqueness of nondecaying solutions for the Navier-Stokes equations, 19 pages. 2002.

\#561 M. Jinzenji and T. Sasaki, An Approach to $\mathcal{N}=4 A D E$ gauge Theory on $K 3,29$ pages. 2002.

\#562 T. Nakazi and T. Yamamoto, Norms of some singular integral operators on weighted $L^{2}$ spaces, 27 pages. 2002 .

\#563 A. Harris and Y. Tonegawa, A $\partial \bar{\partial}$-poincaré lemma for forms near an isolated complex singularity, 8 pages. 2002.

\#564 M. Takahashi, Bifurcations of ordinary differential equations of Clairaut type, 23 pages. 2002.

\#565 G. Ishikawa, Classifying singular Legendre curves by contactomorphisms, 17 pages. 2002.

\#566 G. Ishikawa, Perturbations of Caustics and fronts, 17 pages. 2002.

\#567 Y. Giga, On regularizing-decay rate estmates for solutions to the Navier-Stokes initial value problem, 12 pages. 2002.

\#568 T. Miyao, Strongry supercommuting serf-adjoint operators, 34 pages. 2002.

\#569 Jun-Muk Hwang and K. Yamaguchi, Characterization of Hermitian symmetric spaces by fundamental forms, 10 pages. 2002.

\#570 H. Ishii and T. Mikami, Convexified Gauss curvature flow of bounded open sets in an anisotropic external field: a stochastic apporoximation and PDE, 37 pages. 2002.

\#571 Y. Nakano, Minimization of shortfall risk in a jump-diffusion model, 10 pages. 2002.

\#572 K. Izuchi and T. Nakazi, Backward Shift Invariant Subspaces in the Bidisc, 8 pages. 2002. 


\title{
The horospherical geometry of surfaces in Hyperbolic 4-space
}

\author{
S. Izumiya ${ }^{*}$ D. Pei ${ }^{\dagger}$ and M.C. Romero-Fuster ${ }^{\ddagger}$
}

\begin{abstract}
We study some geometrical properties associated to the contacts of surfaces with hyperhorospheres in $H_{+}^{4}(-1)$. We introduce the concepts of osculating hyperhorospheres, horobinormals, horoasymptotic directions and horospherical points and provide conditions ensuring their existence. We show that totally semiumbilical surfaces have orthogonal horoasymptotic directions.
\end{abstract}

\section{Introduction}

A hypersurface given by the intersection of the hyperbolic $n$-space $H_{+}^{n}(-1)$ with a spacelike, timelike or lightlike hyperplane of $\mathbb{R}_{1}^{n+1}$ is respectively called $h y$ persphere, equidistant hyperplane or hyperhorosphere. The last ones have the curious property of inheriting a euclidean geometry as submanifolds of the hyperbolic space. A great deal of the properties of submanifolds of euclidean spaces can be studied at the light of their contacts with invariant subsets, such as hyperplanes or hyperspheres, of the ambient space (see for instance, [7], [8] or [9]). In a similar way, given any submanifold $M \subset H_{+}^{n}(-1)$, the study of its contacts with hyperhorospheres leads to certain properties that give rise to the horospherical geometry of $M$. An introduction to this for hypersurfaces in $H_{+}^{n}(-1)$ has been given in [3]. On the other hand, the geometry associated to the contacts of surfaces with lightlike hyperplanes in $\mathbb{R}_{1}^{4}$ has been described in [4] through the analysis of the singularities of the lightcone height functions family $H: M \times S_{+}^{2} \rightarrow \mathbb{R}$. These are tightly related to those of the lightcone Gauss map. The contacts that concern us here, namely, those of a surface

*Work partially supported by Grant-in-Aid for Scientific Research, JSPS, No.12874007

†Work partially supported by Grant-in-Aid for Scientific Research, JSPS, No.12000266

Work partially supported by DGCYT grant no. BFM2000-1110 
$M=g(U) \subset H_{+}^{4}(-1)$ with hyperhorospheres can be similarly described by means of the lightcone height functions family $H: M \times S_{+}^{3} \rightarrow \mathbb{R}$. This setting allows us to see that $M$ may have at some points a stronger contact with certain hyperhorospheres, that we call osculating hyperhorospheres. They play an equivalent role to that of osculating hyperplanes of surfaces immersed in euclidean 4-space introduced in [7]. We notice that there is an essential difference: whereas in the euclidean case the maximum number of osculating hyperplanes at a point of a generic surface is 2 , we show in our case that there may be up to four osculating hyperhorospheres at each point of a generic surface immersed in $H_{+}^{4}(-1)$ (Proposition 4.4). We define the horospherical points as those at which some osculating hyperhorosphere has a contact of corank 2 with the surface. They are the analogous of the inflection points in the euclidean case. We show in Proposition 4.6 that the curvature ellipse at these points is degenerate (i. e., they are semiumbilic, see [5]). We characterize them as critical points of certain direction fields on $M$ that we introduce and call horoasymptotic here. We obtain some conditions guaranteeing the global existence of such fields on the surface (Theorem 5.2) and from these that of horospherical points (Corollary 5.3). This leads us to put forward the following horospherical version of Caratheodory's conjecture:

Any 2-sphere immersed as an everywhere horohyperbolic surface in hyperbolic 4-space has at least 2 horospherical points.

Finally, we show that, provided the horoasymptotic fields are globally defined on $M$, total semiumbilicity implies orthogonality of their integral lines (theorem $5.4)$.

\section{Basic concepts and notations}

We consider the $(n+1)$-dimensional Minkowski space $\left(\mathbb{R}^{n+1},\langle\rangle,\right)$, with the pseudo scalar product given by $\left\langle\left(x_{1}, x_{2}, \ldots, x_{n+1}\right),\left(y_{1}, y_{2}, \ldots, y_{n+1}\right)\right\rangle=-x_{1} y_{1}+$ $x_{2} y_{2}+\ldots+x_{n+1} y_{n+1}$. We shall denote this space by $\mathbb{R}_{1}^{n+1}$.

We say that a vector $\mathbf{x}=\left(x_{1}, \ldots, x_{n+1}\right) \in \mathbb{R}_{1}^{n+1} \backslash\{\mathbf{0}\}$ is spacelike, timelike or lightlike provided $\langle\mathbf{x}, \mathbf{x}\rangle>0,=0$ or $<0$ respectively. The norm or length of a vector $\mathbf{x} \in \mathbb{R}_{1}^{4}$ is defined by $\|\mathbf{x}\|=(|\langle\mathbf{x}, \mathbf{x}\rangle|)^{\frac{1}{2}}$.

Given a vector $\mathbf{v} \in \mathbb{R}_{1}^{n+1}$ and a real number $c$, we define the hyperplane with pseudonormal $\mathbf{v}$ as

$$
H P(\mathbf{v}, c)=\left\{\mathbf{x} \in \mathbb{R}_{1}^{n+1} \mid\langle\mathbf{x}, \mathbf{v}\rangle=c\right\} .
$$

This hyperplane is said to be spacelike, timelike or lightlike according to $\mathbf{v}$ is timelike, spacelike or lightlike.

We define the Hyperbolic n-space by

$$
H_{+}^{n}(-1)=\left\{\mathrm{x} \in \mathbb{R}_{1}^{n+1} \mid\langle\mathbf{x}, \mathbf{x}\rangle=-1, x_{1} \geq 1\right\}
$$


The hypersurfaces of $H_{+}^{n}(-1)$ determined by intersecting it with a spacelike, a timelike or a lightlike hyperplane are respectively called hyperspheres, equidistant hyperplanes and hyperhorospheres.

We also consider the set

$$
L C_{\mathbf{a}}=\left\{\mathbf{x} \in \mathbb{R}_{1}^{n+1} \mid\langle\mathbf{x}-\mathbf{a}, \mathbf{x}-\mathbf{a}\rangle=0\right\}
$$

known as the $n$-dimensional lightcone with vertex $\mathbf{a} \in \mathbb{R}_{1}^{n+1}$. The subset,

$$
S_{+}^{n-1}(\mathbf{a})=\left\{\mathbf{x}=\left(x_{1}, x_{2}, \ldots, x_{n+1}\right) \mid\langle\mathbf{x}-\mathbf{a}, \mathbf{x}-\mathbf{a}\rangle=0, x_{1}=a_{1}+1\right\}
$$

is the lightcone $(n-1)$-sphere centered at $\mathbf{a}=\left(a_{1}, a_{2}, \ldots, a_{n+1}\right)$. It shall be denoted as $S_{+}^{n-1}$ when centered at the origin.

Suppose that $M$ is a surface immersed in $\mathbb{R}_{1}^{n+1}$. We say that $M$ is a spacelike surface if the tangent plane $T_{\mathrm{x}} M$ is spacelike (i.e., consists of spacelike vectors) and thus a euclidean plane $\left(T_{\mathbf{x}} M,\langle\rangle,\right)$ for every point $\mathrm{x} \in M$. In this case, the normal space $N_{\mathrm{x}} M$ is a Lorentz $(n-1)$-space $\left(\left(N_{\mathrm{x}} M,\langle\rangle,\right)\right.$.

\section{Second fundamental form and curvature el- lipses}

Given a smooth oriented surface $M$ immersed in $\mathbb{R}_{1}^{5}$, we denote by $\mathcal{X}(M)$ and $\mathcal{N}(M)$ the space of the smooth vector fields tangent to $M$ and the space of the smooth vector fields normal to $M$, respectively. Consider the second fundamental map,

$$
\alpha: \mathcal{X}(M) \times \mathcal{X}(M) \rightarrow \mathcal{N}(M), \alpha(X, Y)=\bar{\nabla}_{\bar{X}} \bar{Y}-\nabla_{X} Y
$$

where $\bar{\nabla}$ denotes the pseudoriemannian connection of $\mathbb{R}_{1}^{5}$ and $\bar{X}$ and $\bar{Y}$ are local extensions to $\mathbb{R}_{1}^{5}$ of tangent vector fields $X$ and $Y$ on $M$. This map is well defined, symmetric and bilinear. Given any normal field $\nu \in \mathcal{N}(M) \backslash\{0\}$, we have for each $\mathrm{x} \in M$ a function

$$
H_{\nu}: T_{\mathbf{x}} M \times T_{\mathbf{x}} M \rightarrow \mathbb{R}, H_{\nu}(\mathbf{v}, \mathbf{w})=\langle\alpha(\mathbf{v}, \mathbf{w}), \nu(\mathbf{x})\rangle .
$$

which is also symmetric and bilinear. The second fundamental form of $M$ at $\mathbf{x}$ is the associated quadratic form,

$$
\left.I I_{\nu}: T_{\mathbf{x}} M \rightarrow \mathbb{R}, I I_{\nu}()=H_{\nu} \mathbf{v}, \mathbf{v}\right)
$$

Suppose that $M$ is locally defined at $\mathrm{x}$ by $g: \mathbb{R}^{2} \rightarrow \mathbb{R}_{1}^{5}$ such that $g(0,0)=\mathbf{x}$ and choose local isothermic coordinates $\{x, y\}$ on $M$ and a pseudo-orthonormal frame, $\left\{e_{1}, e_{2}, e_{3}, e_{4}, e_{5}\right\}$ in a neighbourhood of $\mathbf{x}=g(0,0) \in M$, such that $\left\{e_{1}, e_{2}, e_{3}\right\}$ is a normal frame and $\left\{e_{4}, e_{5}\right\}$ a tangent frame, with $\left\langle e_{1}, e_{1}\right\rangle=-1$ 
and $\left\langle e_{i}, e_{i}\right\rangle=1, i=2, \ldots, 5$. Then the matrix of the bilinear form $H_{\boldsymbol{e}_{i}}$ is given by

$$
H_{e_{i}}(\mathbf{x})=\left[\begin{array}{cc}
a_{i} & b_{i} \\
b_{i} & c_{i}
\end{array}\right],
$$

where if $d s^{2}=E\left(d x^{2}+d y^{2}\right)$ is the first fundamental form, we have

$$
a_{i}=\frac{1}{E}\left\langle\frac{\partial^{2} g}{\partial x^{2}}(0,0), e_{i}\right\rangle, \quad b_{i}=\frac{1}{E}\left\langle\frac{\partial^{2} g}{\partial x \partial y}(0,0), e_{i}\right\rangle, \quad c_{i}=\frac{1}{E}\left\langle\frac{\partial^{2} g}{\partial y^{2}}(0,0), e_{i}\right\rangle
$$

for $i=1,2,3$.

Given $\mathrm{x} \in M$, consider the linear map induced by the second fundamental form on $M, A_{\mathbf{x}}: N_{\mathbf{x}} M \rightarrow Q^{2}$, where $Q^{2}$ is the space of quadratic forms in two variables. That is, $A_{\mathbf{x}}(\mathbf{v})=I I_{\mathbf{v}}, \forall \mathbf{v} \in N_{\mathbf{x}} M$.

In the same coordinates as above, we have that if $\mathbf{v}=v_{1} e_{1}+v_{2} e_{2}+v_{3} e_{3}$, then

$$
A_{\mathbf{x}}(\mathbf{v})=\frac{1}{E}\left(v_{1}\left\langle d^{2} g(0,0), e_{1}\right\rangle+v_{2}\left\langle d^{2} g(0,0), e_{2}\right\rangle+v_{3}\left\langle d^{2} g(0,0), e_{3}\right\rangle\right)
$$

and thus the matrix of $A_{\mathbf{x}}$ is

$$
\left[\begin{array}{lll}
a_{1} & b_{1} & c_{1} \\
a_{2} & b_{2} & c_{2} \\
a_{3} & b_{3} & c_{3}
\end{array}\right] .
$$

We say that a point $\mathbf{x} \in M$ into the type $M_{i}, i=3,2,1,0$ provided $\operatorname{rank} A_{\mathbf{x}}=$ $i$. It was shown in ([8], Propositions 2 and 3 ) that for generic surfaces in euclidean 5-space the $M_{3}$-points fill an open and dense submanifold, whereas the $M_{2}$ points form closed regular curves and the $M_{1}$ and $M_{0}$ points can be avoided. We remark that the arguments there used can be easily adapted to the case that concerns us here, so that we can conclude that the same assertions hold for generic spacelike surfaces immersed in 5-dimensional Minkowski space.

Given $\mathbf{x} \in M$, consider the unit circle in $T_{\mathbf{x}} M$ parametrized by the angle $\theta \in[0,2 \pi]$. Denote by $\gamma_{\theta}$ the spacelike curve obtained by intersecting $M$ with the timelike hyperplane defined by the direct sum of the normal subspace $N_{\mathbf{x}} M$ and the straight line in the tangent direction represented by $\theta$. The curvature vector $\eta(\theta)$ of $\gamma_{\theta}$ in $\mathbf{x}$ lies in the timelike hyperplane $N_{\mathbf{x}} M$. Varying $\theta$ from 0 to $2 \pi$, the vector $\eta(\theta)$ describes an ellipse in $N_{\mathrm{x}} M$, called the curvature ellipse of $M$ at $\mathbf{x}$. This ellipse is the image of the affine map (see [5] for the case $n=3$, the case $n \geq 4$ is a straightforward generalization)

$$
\eta: S^{1} \subset T_{\mathbf{x}} M \longrightarrow N_{\mathbf{x}} M
$$


given by

$$
\theta \longmapsto \eta(\theta)=\sum_{i=1}^{3}\left[\begin{array}{ll}
\cos \theta & \sin \theta
\end{array}\right] \cdot\left[\begin{array}{ll}
a_{i} & b_{i} \\
b_{i} & c_{i}
\end{array}\right] \cdot\left[\begin{array}{c}
\cos \theta \\
\sin \theta
\end{array}\right] \cdot e_{i},
$$

that is,

$$
\eta(\theta)=H_{\mathbf{x}}+B_{\mathbf{x}} \cos 2 \theta+C_{\mathbf{x}} \sin 2 \theta,
$$

with

$$
\begin{gathered}
H_{\mathrm{x}}=\frac{1}{2}\left(a_{1}+c_{1}\right) e_{1}-\frac{1}{2} \sum_{i=2}^{3}\left(a_{i}+c_{i}\right) \cdot e_{i}, \\
B_{\mathbf{x}}=\frac{1}{2}\left(a_{1}-c_{1}\right) e_{1}-\frac{1}{2} \sum_{i=2}^{3}\left(a_{i}-c_{i}\right) \cdot e_{i}, \\
C_{\mathbf{x}}=b_{1} e_{1}-\sum_{i=2}^{3} b_{i} \cdot e_{i} .
\end{gathered}
$$

Lemma 3.1 Given a spacelike surface $M \subset \mathbb{R}_{1}^{5}$, the subspace $\operatorname{Ker} A_{\mathrm{x}}$ determined by the kernel of $A_{\mathbf{x}}$ in $N_{\mathbf{x}} M$ is pseudo-orthogonal to the vectors $B_{\mathbf{x}}$ and $C_{\mathrm{x}}$ that define the curvature ellipse.

Proof: Suppose that $\mathbf{x} \in M_{i}, i<3$, for otherwise $\operatorname{Ker} A_{\mathbf{x}}=\{0\}$ and the result is trivial. Given $\mathbf{v}=v_{1} e_{1}+v_{2} e_{2}+v_{3} e_{3} \in \operatorname{Ker} A_{\mathbf{x}}$, we have that $a_{1} v_{1}+a_{2} v_{2}+a_{3} v_{3}=$ $b_{1} v_{1}+b_{2} v_{2}+b_{3} v_{3}=c_{1} v_{1}+c_{2} v_{2}+c_{3} v_{3}=0$. Then $2\left\langle\mathbf{v}, B_{\mathbf{x}}\right\rangle=-v_{1}\left(a_{1}-c_{1}\right)-$ $v_{2}\left(a_{2}-c_{2}\right)-v_{3}\left(a_{3}-c_{3}\right)=0$ and $\left\langle\mathbf{v}, C_{\mathbf{x}}\right\rangle=-v_{1} b_{1}-v_{2} b_{2}-v_{3} b_{3}=0 \square$

The curvature ellipse at $\mathbf{x}$ is contained in the Lorentz 3 -space $N_{\mathbf{x}} M$ and it may be degenerate (a segment or even a point) at certain points of $\mathrm{x} \in M$ that are called semiumbilics. A semiumbilic point $\mathbf{x}$ is said to be spacelike, timelike or lightlike provided the curvature segment defines respectively a spacelike, timelike or lightlike direction in $N_{\mathbf{x}} M$. The points at which the curvature ellipse becomes a point are degenerate semiumbilics known as umbilics. It is a straightforward exercise to verify that any semiumbilic point is of type $M_{i}, i<3$. We notice that although $M_{1}$ points are either semiumbilic or umbilic, not every point of type $M_{2}$ needs to be a semiumbilic. Moreover, it was shown in [10] that the semiumbilics of generically immersed surfaces in euclidean 5-space are isolated points (lying on curves of $M_{2}$ - points) and it is not difficult to see that similar arguments apply to the case of surfaces generically immersed in Minkowski 5-space. A surface all whose points are semiumbilic is said to be totally semiumbilical. Some of the properties of totally semiumbilical surfaces in $\mathbb{R}_{1}^{n+1}$ were studied in [5]. In particular, for surfaces contained in hyperbolic 4-space we have, 
Proposition 3.2 ([5]) Given a surface $M \subset H_{+}^{4}(-1)$, the curvature ellipse of $M$ at a point $\mathbf{x} \in M$ is contained in an affine plane of $N_{\mathbf{x}} M$ parallel to $T_{\mathbf{x}} H_{+}^{4}(-1) \cap N_{\mathbf{x}} M$.

We shall see as a consequence of this, in the next section, that the generic behaviour of semiumbilic points of surfaces contained in $H_{+}^{4}(-1)$ differs from the case of surfaces in $\mathbb{R}_{1}^{5}$.

The shape operator associated to a normal field $\nu$ is defined as

$$
S_{\nu}: T M \rightarrow T M, S_{\nu}(X)=-\left(\bar{\nabla}_{\bar{X}} \bar{\nu}\right)^{\top},
$$

where $\bar{\nu}$ is a local extension to $\mathbb{R}_{1}^{5}$ of the normal vector field $\nu$ at $\mathbf{x}$ and ()$^{\top}$ means the tangent component. This operator is bilinear, self-adjoint and satisfies the following equation: $\left\langle S_{\nu}(X), Y\right\rangle=H_{\nu}(X, Y), \forall X, Y \in \mathcal{X}(M)$. So, we have that $I I_{\nu}(X)=\left\langle S_{\nu}(X), X\right\rangle$.

We can find for each $\mathrm{x} \in M$, an orthonormal basis of $T_{\mathrm{x}} M$ consisting of eigenvectors of $S_{\nu}$, for which the restriction of the second fundamental form to the unitary vectors, $\left.I I_{\nu}\right|_{S^{1}}$, takes its maximal and minimal values. The corresponding eigenvalues $k_{1}, k_{2}$ are the $\nu$-principal curvatures, respectively. A point $\mathbf{x}$ is said to be $\nu$-umbilic if both $\nu$-principal curvatures coincide at $\mathbf{x}$. Let $\mathcal{U}_{\nu}$ be the set of $\nu$-umbilics in $M$. For any $\mathrm{x} \in M \backslash \mathcal{U}_{\nu}$ there are two $\nu$-principal directions defined by the eigenvectors of $S_{\nu}$, these fields of directions are smooth and integrable, then they define two families of orthogonal curves, its integrals, which are called the $\nu$-principal lines of curvature. The two orthogonal foliations with the $\nu$-umbilics as its singularities form the $\nu$-principal configuration of $M$. We say that the surface $M$ is $\nu$-umbilical if each point of $M$ is $\nu$-umbilic. Some umbilicity properties of surfaces immersed in Minkowski spaces have been studied in [5]. It was there proved (Proposition 5.1) that a point $\mathbf{x}$ of a surface $M \subset \mathbb{R}_{1}^{5}$ is $\nu$-umbilic for some normal field $\nu$ if and only if $\nu(\mathbf{x})$ is pseudoorthogonal to the vectors $B_{\mathbf{x}}$ and $C_{\mathbf{x}}$ that define the curvature ellipse at $\mathbf{x}$. In particular, in the case of a surface $M \subset H_{+}^{4}(-1)$ we have that in virtue of Proposition 3.2 as follows:

If $\rho(\mathbf{x})=\mathbf{x}$ is the position (normal) field on $M$, then each point of $M$ is $\rho$-umbilical.

Moreover,

Proposition 3.3 ([5]) A surface $M \subset H_{+}^{4}(-1)$ is totally semiumbilical if and only if $M$ is umbilical with respect to some lightlike normal field.

We also have

Corollary 3.4 ([5]) A surface $M \subset H_{+}^{n}(-1)$ lies in a hyperhorosphere if and only if it is umbilical with respect to some lightlike normal field $\nu$ with constant zero curvature. 


\section{Contacts with lightlike hyperplanes and hy- perhorospheres}

Given submanifolds $M$ and $N$ of $\mathbb{R}^{n+1}$, locally defined by $M=g\left(\mathbb{R}^{m}\right)$ and $N=f^{-1}(0)$, where $g: \mathbb{R}^{m} \rightarrow \mathbb{R}^{n+1}$ is an embedding, and $f: \mathbb{R}^{n+1} \rightarrow \mathbb{R}^{q}$ is a submersion, we can "measure their contact at a common point $p \in M \cap N$ by analyzing the singularities of the composed map $f \circ g: \mathbb{R}^{m} \rightarrow \mathbb{R}^{q}$ ("contact map"). In fact, we say that two submanifolds $M$ and $N$ of a manifold $Z$ have the same contact at a point $p$ as two submanifolds $M^{\prime}$ and $N^{\prime}$ of a manifold $Z^{\prime}$ at a point $p^{\prime}$ if and only if there is a diffeomorphism germ $\phi:(Z, p) \rightarrow\left(Z^{\prime}, p^{\prime}\right)$ taking $M$ to $M^{\prime}$ and $N$ to $N^{\prime}$. In this case we write, $K(M, N)=K\left(M^{\prime}, N^{\prime}\right)$. J.A. Montaldi proved [9] that this holds if and only if their respective contact mapgerms are $\mathcal{K}$-equivalent, where we say that two map-germs $f_{i}:\left(\mathbb{R}^{m}, x_{i}\right) \rightarrow$ $\left(\mathbb{R}^{p}, y_{i}\right), i=1,2$ are contact-equivalent or $\mathcal{K}$-equivalent (denoted $\mathcal{K}\left(f_{1}\right)=$ $\left.\mathcal{K}\left(f_{2}\right)\right)$ if there is a diffeomorphism-germ (contact-equivalence), $H:\left(\mathbb{R}^{m} \times\right.$ $\left.\mathbb{R}^{p},\left(x_{1}, y_{1}\right)\right) \rightarrow\left(\mathbb{R}^{m} \times \mathbb{R}^{p},\left(x_{2}, y_{2}\right)\right)$ of the form $H(x, t)=(h(x), \theta(x, t))$, such that $H\left(x, y_{1}\right)=\left(h(x), y_{2}\right)$ and $H\left(x, f_{1}(x)\right)=\left(h(x), f_{2}(h(x))\right)$. We refer to [2] or [6] for the definition and details on $\mathcal{K}$-equivalence.

Therefore, to study the contact of a spacelike surface locally given as $M=$ $g\left(\mathbb{R}^{2}\right) \subset \mathbb{R}_{1}^{5}$ with a hyperplane $H(\mathbf{v}, \mathbf{x})=H(\mathbf{v},\langle\mathbf{x}, \mathbf{v}\rangle)$ at a point $\mathbf{x}=g(u) \in$ $M \cap H$, the map $f$ has to be chosen in such a way that $H(\mathbf{v}, \mathbf{x})=f^{-1}(0)$, that is,

$$
f\left(x_{1}, \ldots, x_{5}\right)=-x_{1} \cdot v_{1}+\ldots+x_{5} \cdot v_{5}-\langle\mathbf{x}, \mathbf{v}\rangle,
$$

where $\mathbf{v}=\left(v_{1}, \ldots, v_{5}\right)$ is a pseudonormal vector to $H(\mathbf{v}, \mathbf{x})$.

And hence, to analyze all the possible contacts of the submanifold $M=$ $g\left(\mathbb{R}^{2}\right)$ with the lightlike hyperplanes of $\mathbb{R}^{5}$, we must describe the singularities of the lightcone height functions family

$$
\begin{aligned}
H: \mathbb{R}^{2} \times S_{+}^{3} & \longrightarrow \mathbb{R} \\
(u, \mathbf{v}) & \longmapsto<g(u), \mathbf{v}\rangle
\end{aligned}
$$

We shall denote by $h_{\mathbf{v}}$ the function obtained when fixing the parameter $\mathbf{v}$. Clearly, $u$ is a singular point of $h_{\mathbf{v}}$ if and only if $\mathbf{v} \in N_{\mathbf{X}(u)} M$.

Suppose now that $M$ lies in $H_{+}^{4}(-1)$. Given $\mathbf{v} \in S_{+}^{3}$ and $\mathbf{x} \in M$, let us denote by $\Omega(\mathbf{v}, \mathbf{x})$ the hyperhorosphere determined on $H_{+}^{4}(-1)$ by the lightlike hyperplane with pseudo-normal $\mathbf{v}$ passing trough the point $\mathbf{x}=g(u), H(\mathbf{v}, \mathbf{x})$. We have that $\Omega(\mathbf{v}, \mathbf{x})$ is tangent to $M$ at $\mathbf{x}$ if and only if $u$ is a singular point of $h_{\mathbf{v}}$. Furthermore, 
Lemma 4.1 Given a surface $M=g\left(\mathbb{R}^{2}\right) \subset H_{+}^{4}(-1)$ and $\mathbf{x} \in M$, the contact map-germs of the pairs $(M, \Omega(\mathbf{v}, \mathbf{x}))$ and $(M, H(\mathbf{v}, \mathbf{x}))$ at $\mathbf{x}$ coincide.

Proof: We have that $H(\mathbf{v}, \mathbf{x})=h_{(\mathbf{v}, \mathbf{x})}^{-1}(0)$, where $h_{(\mathbf{v}, \mathbf{x})}: \mathbb{R}_{1}^{5} \rightarrow \mathbb{R}$ is given by $h_{(\mathbf{v}, \mathbf{x})}(\mathbf{p})=\langle\mathbf{v}, \mathbf{p}\rangle-\langle\mathbf{v}, \mathbf{x}\rangle$. So, if we represent by $i: H_{+}^{4}(-1) \rightarrow \mathbb{R}_{1}^{5}$ the canonical inclusion, we have that the contact map for $H(\mathbf{v}, \mathbf{x})$ and $M$ is given by $h_{(\mathbf{v}, \mathbf{x})} \circ i \circ g$. On the other hand, if we denote $\bar{h}_{(\mathbf{v}, \mathbf{x})}=\left.h_{(\mathbf{v}, \mathbf{x})}\right|_{H_{-}^{4}(-1)}$, we have that $\bar{h}_{(\mathbf{v}, \mathbf{x})}^{-1}(0)=H(\mathbf{v}, \mathbf{x}) \cap H_{+}^{4}(-1)=\Omega(\mathbf{v}, \mathbf{x})$, and hence $\bar{h}_{(\mathbf{v}, \mathbf{x})} \circ g$ is the contact map for $M$ and $\Omega(\mathbf{v}, \mathbf{x})$. But, clearly, $\bar{h}_{(\mathbf{v}, \mathbf{x})} \circ g=h_{(\mathbf{v}, \mathbf{x})} \circ i \circ g$.

Given a singular point $u$ of the function $h_{\mathbf{v}}$, if the hessian matrix $H e s s h_{\mathbf{v}}(u)$ defines a degenerate quadratic form we say that $\mathbf{v}$ is a horobinormal direction for $M$ at $\mathbf{x}=g(u)$. In this case we have that $\Omega(\mathbf{v}, \mathbf{x})$ has higher order contact with $M$ at $\mathrm{x}$ and we call it osculating hyperhorosphere. A normal field $\nu$ defined on some open subset $V$ of $M$ and such that $\nu(\mathbf{x})$ is a horobinormal direction at $\mathbf{x}, \forall \mathbf{x} \in V$ is called a horobinormal field on $V$.

Given $\mathrm{x} \in M$, consider the linear map $A_{\mathrm{x}}: N_{\mathrm{x}} M \rightarrow Q^{2}$ and denote by $C$ the cone of degenerate quadratic forms in $Q^{2}$. We observe that $\mathbf{v} \in N_{\mathbf{x}} M$ determines a horobinormal if and only if $\mathbf{v} \in A_{\mathbf{x}}^{-1}(C) \cap L C_{\mathbf{x}}$.

A particular feature of the spacelike surfaces contained in hyperbolic 4-space is the following.

Lemma 4.2 The points of type $M_{2}$ of a surface $M \subset H_{+}^{4}(-1)$ are all semiumbilic.

Proof: Take a point $\mathrm{x} \in M$ of type $M_{2}$ and suppose that $M$ is locally defined at $\mathbf{x}$ by an embedding $g: \mathbb{R}^{2} \rightarrow H_{+}^{4}(-1)$, such that $\mathbf{x}=g(0,0)$. Then the height function $h_{\mathbf{x}}(u)=\langle g(u), \mathbf{x}\rangle-\langle g(0,0), \mathbf{x}\rangle=\langle g(u), \mathbf{x}\rangle+1$ describes the contact of $M$ at $\mathbf{x}$ with the hyperplane $H_{\mathrm{x}}$ pseudo-orthogonal to the vector $\mathbf{x}$. Observe that if we take $g$ in the Monge form $g(u)=\left(u, g_{1}(u), g_{2}(u), g_{3}(u)\right)$, it is not difficult to verify that the hessian matrix of $h_{\mathrm{x}}$ at $(0,0)$ coincides with that of $A_{\mathrm{x}}(\mathrm{x})$. If we assume now that $\mathrm{x}$ is not semiumbilic, it follows from Lemma 3.1 together with Proposition 3.2 that $\operatorname{Ker} A_{\mathbf{x}}$ is spanned by the position vector $\mathrm{x}$ and hence $\operatorname{Hessh}_{\mathrm{x}}(0,0)$ is the null matrix. This means that $(0,0)$ is a non stable singularity of $h_{\mathbf{x}}$, which implies that the extension of this function to $H_{+}^{4}(-1)$ also has a non stable singularity at $\mathrm{x} \in H_{+}^{4}(-1)$. But it can be seen that the contacts of $H_{+}^{4}(-1)$ with all its tangent hyperplanes are non degenerate in the sense that they have the minimal possible order, or in other words, they lead to a stable height function. So we arrive to a contradiction.

This leads to the following result concerning the distribution of semiumbilic points over surfaces generically immersed in $H_{+}^{4}(-1)$, which supposes an interesting difference with respect to the generic behaviour of surfaces immersed in Minkowski 5-space. 
Proposition 4.3 Given a surface $M$ generically immersed in $H_{+}^{4}(-1)$, the points of type $M_{3}$ fill an open and dense submanifold and the semiumbilic points are all of type $M_{2}$ and define a closed curves embedded in $M$. Umbilic points do not appear on these surfaces.

Proof: Let $\Delta(\mathbf{x})=\operatorname{det} A_{\mathrm{x}}$. It is clear that $\Delta^{-1}(0)=M-M_{3}$. Since $\Delta$ is a continuous function on $M$, we have that $M_{3}$ must be an open region in $M$. The condition that $\mathrm{x} \in M_{2}$ implies by Lemma 4.2 that $\mathrm{x}$ is semiumbilic. But this is equivalent to requiring that the normal vectors $B_{\mathbf{x}}$ and $C_{\mathbf{x}}$ be linearly dependent. Since by Proposition 3.2 we know that $B_{\mathbf{x}}, C_{\mathbf{x}} \in T_{\mathbf{x}} H_{+}^{4}(-1)$, it follows that the position vector $\mathbf{x}$ must be pseudo-orthogonal to both $B_{\mathbf{x}}$ and $C_{\mathbf{x}}$. We can give $M$ by a local embedding $g: \mathbb{R}^{2} \rightarrow H_{+}^{4}(-1) \subset \mathbb{R}_{1}^{5}$ in the Monge form at $\mathbf{x}$ and take a pseudo-orthonormal frame $\left\{e_{1}, e_{2}, e_{3}, e_{4}, e_{5}\right\}$ for $M$ in a neighbourhood of the point $\mathbf{x}$ in such a way that $e_{1}$ is the position vectorfield, $e_{2}$ and $e_{3}$ are normal vectorfields and $e_{4}, e_{5}$ generate the tangent planes. In these coordinates, we can write $B_{\mathrm{x}}=-\frac{1}{2}\left(a_{2}-c_{2}\right) \cdot e_{2}-\frac{1}{2}\left(a_{3}-c_{3}\right) \cdot e_{3}$ and $C_{\mathbf{x}}=-b_{2} \cdot e_{2}-b_{3} \cdot e_{3}$. Then the linearly dependence of these two vectors is given by the requirement

$$
\frac{a_{2}-c_{2}}{b_{2}}=\frac{a_{3}-c_{3}}{b_{3}},
$$

which defines a 1-codimensional algebraic variety of the jet space $J^{2}\left(\mathbb{R}^{2}, \mathbb{R}^{5}\right)$. It follows now from the Thom Transversality Theorem ([2]) that the 2-jet extension, $j^{2} g: \mathbb{R}^{2} \rightarrow J^{2}\left(\mathbb{R}^{2}, \mathbb{R}^{5}\right)$, meets this submanifold transversally and therefore, the considered points determine an algebraic subset of codimension 1 in $M$.

On the other hand, the condition that $\mathrm{x} \in M_{1} \cup M_{0}$ is equivalent to asking that $\operatorname{rank} A_{\mathbf{x}} \leq 1$. This means that the vector $H_{\mathbf{x}}$ must also be parallel to both $B_{\mathrm{x}}$ and $C_{\mathrm{x}}$. This provides at least three independent quadratic equations in $J^{2}\left(\mathbb{R}^{2}, \mathbb{R}^{5}\right)$ and by using again Thom Transversality Theorem we can conclude that, for a generic $g, j^{2} g$ does not meet the corresponding algebraic variety, and thus $M_{1} \cup M_{0}=\emptyset$.

So $\Delta^{-1}(0)=M_{2}$ is completely made of semiumbilic points. We see now that they form embedded curves. In fact, let

$$
\begin{aligned}
g: \mathbb{R}^{2}, 0 & \longrightarrow \mathbb{R}_{1}^{5} \\
(u, v) & \longmapsto\left(u, v, g_{1}(u, v), g_{2}(u, v), g_{3}(u, v)\right)
\end{aligned}
$$

be the local representation of $M$ in the Monge form at $\mathrm{x} \in M_{2}$. In these coordinates $\Delta(\mathbf{x})=g_{1 u u} g_{2 u v} g_{3 v v}-g_{1 u v} g_{2 u u} g_{3 v v}-g_{1 u u} g_{2 v v} g_{3 u v}+g_{1 v v} g_{2 u u} g_{3 u v}+$ $g_{1 u v} f_{2 v v} f_{3 u u}-f_{1 v v} f_{2 u v} f_{3 u u}$. It follows from this expression that, under appropriate transversality conditions on the 3 -jet of $g$, the set $\Delta=0$ represents a curve possibly with isolated singular points determined by the vanishing of the derivatives of the function $\Delta$. We observe that the pseudo-orthogonality property of the frame $\left\{e_{1}, e_{2}, e_{3}, e_{4}, e_{5}\right\}$ is irrelevant for our study. For a change of 
basis in $N_{\mathbf{x}} M$ preserves the relative position of $\operatorname{Im}\left(A_{\mathrm{x}}\right)$ with the cone $C$ in $Q^{2}$, and thus the sets $M_{3}$ and $M_{2}$. So we can take $\left\{e_{1}, e_{2}, e_{3}\right\}$ such that $e_{1}$ generates $\operatorname{Ker}\left(A_{\mathbf{x}}\right)$.

If $p \in M_{2}$, we have three possibilities:

i) $\operatorname{Im}\left(A_{\mathbf{x}}\right) \cap C$ is a couple of lines,

ii) $\operatorname{Im}\left(A_{\mathbf{x}}\right) \cap C$ is a line, and

iii) $\operatorname{Im}\left(A_{\mathrm{x}}\right) \cap C$ is just the origin.

In case i) we can choose $\left\{e_{2}, e_{3}\right\}$ as the two (degenerate) directions lying in $A^{-1}(C) \subset N_{p} M$. Furthermore, we can also make a change of coordinates in the source, such that the two degenerate directions correspond to the quadratic forms $u^{2}$ and $v^{2}$ in $C$. Thus $g$ can be locally written as

$$
g(u, v)=\left(u, v, u^{2}+R_{1}(u, v), v^{2}+R_{2}(u, v), R_{3}(u, v)\right),
$$

where $R_{i} \in m^{3}$, i.e., all the derivatives of the $R_{i}$ vanish up to order $3, i=1,2,3$.

In case ii) $\operatorname{Im}\left(A_{\mathbf{x}}\right)$ is tangent to $C$ and we take $e_{3}$ as the generator of $A_{\mathrm{x}}^{-1}\left(\operatorname{Im}\left(A_{\mathrm{x}}\right) \cap C\right)$. With additional change of coordinates in the source, $g$ can be written as

$$
g(u, v)=\left(u, v, u^{2}-v^{2}+R_{1}(u, v), u v+R_{2}(u, v), R_{3}(u, v)\right),
$$

Finally, in case iii), all the quadratic forms $A_{\mathbf{x}}(\mathbf{v}$ are hyperbolic, and $g$ can be written as

$$
g(u, v)=\left(u, v, u^{2}+R_{1}(u, v), u v+R_{2}(u, v), R_{3}(u, v)\right),
$$

In each of the above cases it is a simple (but tedious) calculation to verify that under generic conditions on the 3 -jet of $g$ at $(0,0)$, the derivatives of the function $\Delta$ do not vanish at $\mathrm{x}$ and thus it is a regular point of $\Delta^{-1}(0)$.

We analyse next the possibilities that we may have for the sets $A_{\mathrm{x}}^{-1}(C) \cap L C_{\mathrm{x}}$ at different points $\mathbf{x} \in M_{i}, i=3,2,1,0$ :

a) If $\mathrm{x} \in M_{3}$, then $A_{\mathrm{x}}^{-1}(C)$ is a nondegenerate cone. The intersection $A_{\mathrm{x}}^{-1}(C) \cap L C_{\mathrm{x}}$ depends on the relative position of both cones and may thus consist in either four, three, two, one or no lines in $N_{\mathrm{x}} M$. We remark that it is also possible that both cones $A_{\mathrm{x}}^{-1}(C)$ and $L C_{\mathrm{x}}$ coincide, but this is a extremely degenerate phenomena that can be generically avoided, so we shall not consider it here.

b) If $\mathrm{x} \in M_{2}$, as we have seen before, the plane $\operatorname{Im} A_{\mathrm{x}}$ intersects the cone $C$ in either i) two lines, ii) one line or iii) just the origin. In this case $A_{\mathrm{x}}^{-1}(C)$ is respectively made of i) two planes with the common line $\operatorname{Ker} A_{\mathbf{x}}$, ii) a plane containing the line $\operatorname{Ker} A_{\mathrm{x}}$, or iii) just the line $\operatorname{Ker} A_{\mathrm{x}}$. Again, the intersection $A_{\mathrm{x}}^{-1}(C) \cap L C_{\mathrm{x}}$ depends on the relative position of both subsets and will thus consist in at most four lines in $N_{\mathrm{x}} M$. 
c) When $\mathbf{x} \in M_{1}, \operatorname{Im} A_{\mathbf{x}}$ is a line that may either lie on $C$ or intersect it just at the origin. Correspondingly, $A_{\mathbf{x}}^{-1}(C)$ is the whole normal space $N_{\mathbf{x}} M$, or a plane. It follows that $A_{\mathrm{x}}^{-1}(C) \cap L C_{\mathrm{x}}$ may consist of the whole $L C_{\mathrm{x}}$ in the first case, or at most two lines in the second.

d) Finally, in case $\mathbf{x} \in M_{0}, A_{\mathbf{x}}^{-1}(C)$ is the whole normal space and $A_{\mathbf{x}}^{-1}(C) \cap$ $L C_{\mathbf{x}}=L C_{\mathbf{x}}$.

Therefore, by taking into account that generic surfaces in $H_{+}^{4}(-1)$ are exclusively made of points of types $M_{3}$ and semiumbilics $\left(M_{2}\right)$, we can state the following

Proposition 4.4 The number of osculating hyperhorospheres at any point of a surface $M$ generically immersed in $H_{+}^{4}(-1)$ is at most four.

We say that a point $\mathrm{x} \in M$ of type $M_{3}$ or $M_{2}$ is horoelliptic provided the subsets $A_{\mathrm{x}}^{-1}(C)$ and $L C_{\mathrm{x}}$ intersect only at the origin. On the other hand, it is said to be horohyperbolic or horoparabolic according to they have transversal or nontransversal intersections off the origin. It is not difficult to verify that, generically, horoelliptic and horohyperbolic points determine open submanifolds of $M$ separated by horoparabolic curves. For the particular case of a non semiumbilic $M_{2}$-point $x$ we observe that $x$ is necessarily horohyperbolic, having four horobinormals in case $\mathrm{b}, \mathrm{i}$ ), horoparabolic with two horobinormals in case b,ii) and horoelliptic with no horobinormals in case b,iii). On the other hand, if $x \in M_{2}$ is semiumbilic then both cases, b,i) and b,ii) may correspond to either horohyperbolic, horoparabolic or horoelliptic points. Here we observe that horohyperbolic points may have either four or two horobinormal directions, due to the fact that the line $\operatorname{Ker} A_{x}$ does not need to lie inside the lightcone. Taking into account these considerations, we can conclude

Proposition 4.5 If $M \subset H_{+}^{4}(-1)$ is exclusively made of horohyperbolic points then it has either four or two globally defined horobinormal fields.

It can be shown that, as a consequence of the methods developed by Montaldi ([9]) and analogously to what happens in the case of surfaces generically immersed in Euclidean space, the rank of $\mathrm{Hessh}_{\mathbf{v}}(u)$, for any horobinormal $\mathbf{v}$ at most points $\mathbf{x} \in M$ is 1 . The points at which this rank is 0 are those at which the surface is better approached by the same hyperhorosphere in all the tangent directions. These can be seen as the analogous, in horospherical geometry terms, of the inflection points of surfaces in Euclidean 4-space studied in [1] and shall be called horospherical points.

Proposition 4.6 The horospherical points of a surface $M$ immersed in $H_{+}^{4}(-1)$ are either of semiumbilic or umbilic type. Moreover, every point of type $M_{1}$ or $M_{0}$ is a horospherical point. 
Proof: Suppose that $M$ is given in the Monge form at a horospherical point $\mathbf{x}=g(0,0)$ and take a pseudo-orthonormal frame $\left\{e_{1}, e_{2}, e_{3}, e_{4}, e_{5}\right\}$ in a neighbourhood of $\mathbf{x}$ such that $\left\{e_{4}, e_{5}\right\}$ is a tangent frame and $\left\{e_{1}, e_{2}, e_{3}\right\}$ is a normal frame in this neighbourhood with $\left\langle e_{1}, e_{1}\right\rangle=-1$, as above. Then, for any normal vector $\mathbf{v} \in N_{\mathbf{x}} M$, we can write $\mathbf{v}=v_{1} e_{1}+v_{2} e_{2}+v_{3} e_{3}$. We observe that in this situation the matrices $A_{\mathbf{x}}(\mathbf{v})$ and $\operatorname{Hessh}_{\mathbf{v}}(0,0)$ coincide. Now, under the assumption that $\mathrm{x}$ is horospherical, we can choose a horobinormal vector $\mathbf{v} \in N_{\mathbf{x}} M \cap S_{+}^{3}(\mathbf{x})$, such that all the entries of the matrix $\operatorname{Hessh}_{\mathbf{v}}(0,0)$ vanish, which implies that $I I_{\mathbf{v}}$ also vanishes at $\mathbf{x}$. So we have that $-v_{1} a_{1}+v_{2} a_{2}+v_{3} a_{3}=-v_{1} b_{1}+v_{2} b_{2}+v_{3} b_{3}=-v_{1} c_{1}+v_{2} c_{2}+v_{3} c_{3}=0$, where the $a_{i}, b_{i}, c_{i}, i=1,2,3$ are as in the previous section. But this means that the vectors $B_{\mathbf{x}}$ and $C_{\mathbf{x}}$, that determine the curvature ellipse at $\mathbf{x}$ are both pseudo-orthogonal to the lightlike direction $\mathbf{v}$. On the other hand, it follows from proposition 3.2 that they are also pseudo-orthogonal to the timelike normal direction, $\mathbf{x}$, to $H_{+}^{4}(-1)$ at $\mathbf{x}$. Therefore $\operatorname{rank}\left(B_{\mathbf{x}}, C_{\mathbf{x}}\right) \leq 1$, which implies that the curvature ellipse is degenerated at $\mathbf{x}$, which shows the first assertion. As for the second, we observe that provided $\mathrm{x} \in M_{1}$, we know from Lemma 3.1 that the plane $\operatorname{Ker} A_{\mathrm{x}}$ is pseudo-orthogonal to the direction determined by the (linearly dependent) vectors $B_{\mathrm{x}}$ and $C_{\mathrm{x}}$. But Proposition 3.2 implies that this direction is contained in the plane $T_{\mathbf{x}} H_{+}^{4}(-1)$. Therefore we get that $\operatorname{Ker} A_{\mathbf{x}}$ must contain the line $\langle\mathbf{x}\rangle$ and thus cut the lightcone at $\mathbf{x}$. This determines two horobinormals for which the Hessian of the corresponding lightcone height function has rank 1, and hence $\mathbf{x}$ is a horospherical point. In case $\mathbf{x} \in M_{0}$ we have that all the horobinormals give rise to lightcone height functions whose Hessian has rank 1 at $\mathbf{x}$ and the result also follows.

In particular, we remark that the surfaces contained in a hyperhorosphere of $H_{+}^{4}(-1)$ are a special case of totally semiumbilical surface as can be concluded from Proposition 3.4 and Corollary 3.5.

\section{Horoasymptotic directions}

Given a surface $M$ immersed in $H_{+}^{4}(-1)$, if $\mathbf{v} \in S_{+}^{3}(\mathbf{x})$ is a horobinormal of $M$ at $\mathbf{x}=\mathbf{X}(u)$ we have that $u$ is a degenerate singularity for the height function $h_{\mathbf{v}}$. Here, we consider a local parametrization $\mathbf{X}: U \rightarrow H_{+}^{4}(-1)$ of $M$ at $\mathbf{x}$. Therefore $\operatorname{KerHess}\left(h_{\mathbf{v}}\right)(u) \neq\{0\}$. The non zero directions lying in $\operatorname{KerHess}\left(h_{\mathbf{v}}\right)(u)$ are called horoasymptotic directions at $\mathbf{x}$. We observe that these are the tangent directions at $\mathbf{x}$ along which the higher order contact of $M$ and the hyperhorosphere $\Omega(\mathbf{v}, \mathbf{x})$ occurs. Clearly, any horobinormal field determines a (tangent) horoasymptotic field on the region of $M$ over which it is defined. It follows from the definition of both, horoasymptotic directions and horospherical points, that the last are the critical points of the horoasymptotic fields. We investigate next the possibilities of having some globally defined horoasymptotic field on $M$. 
Lemma 5.1 i) Two horobinormals $\mathbf{b}_{1}$ and $\mathbf{b}_{2}$ at a point $\mathbf{x} \in M$ of type $M_{3}$ cannot share horoasymptotic directions.

ii) Provided $\mathbf{x} \in M$ is of type $M_{i}, i<3$, two horobinormals, $\mathbf{b}_{\mathbf{1}}$ and $\mathbf{b}_{\mathbf{2}}$, share a horoasymptotic direction if and only if they belong to some linear subspace contained in $A_{\mathrm{x}}^{-1}(C)$.

Proof: i) Suppose that $\theta$ is a common asymptotic direction for $\mathbf{b}_{1}$ and $\mathbf{b}_{2}$ at $\mathbf{x}$. In such case we can choose coordinates on $M$ at $\mathbf{x}$ in such a way that

$$
\operatorname{Hess}\left(h_{\mathbf{b}_{\mathbf{1}}}\right)(\mathbf{x})=\left[\begin{array}{cc}
\lambda_{1} & 0 \\
0 & 0
\end{array}\right] \text { and } \operatorname{Hess}\left(h_{\mathbf{b}_{2}}\right)(\mathbf{x})=\left[\begin{array}{cc}
\lambda_{2} & 0 \\
0 & 0
\end{array}\right] \text {. }
$$

But then the normal direction $\mathbf{b}=\lambda_{2} \mathbf{b}_{1}-\lambda_{1} \mathbf{b}_{2}$ has vanishing Hessian matrix at $\mathbf{x}$, which means that $\mathbf{x}$ is a horospherical point. By Proposition 4.3 this implies that $\mathrm{x}$ is a semiumbilic point and these are of type $M_{i}, i<3$ we have arrived to a contradiction.

ii) A similar argument to the used in i) tells us that any common horoasymptotic direction for $\mathbf{b}_{1}$ and $\mathbf{b}_{2}$ at $\mathbf{x}$ lies in the kernel of any linear combination of them. Therefore $\mathbf{b}_{1}$ and $\mathbf{b}_{2}$ define a plane made of degenerate directions of $N_{\mathbf{x}} M$ in the sense that all of them are mapped by $A_{\mathrm{x}}$ into the cone $C$. Conversely, take any $\mathbf{b} \in A_{\mathbf{x}}^{-1}(C)$ that does not belong to $\operatorname{Ker} A_{\mathbf{x}}$ (if all the degenerate directions lie in $\operatorname{Ker} A_{\mathrm{x}}$ we have that all of them share all the tangent directions in $T_{\mathbf{x}} M$ as horoasymptotic directions and the result is trivially true). Then given any other $\mathbf{b}^{\prime}$ lying in the same subspace than $\mathbf{b}$ in $A_{\mathbf{x}}^{-1}(C)$, we can always write $\mathbf{b}^{\prime}=\lambda_{1} \mathbf{b}+\lambda_{2} \mathbf{b}^{\prime \prime}$, for some $\mathbf{b}^{\prime \prime} \in \operatorname{Ker} A_{\mathbf{x}}$ and real numbers $\lambda_{i}, i=1,2$. It is not difficult to see that then $\mathbf{b}$ and $\mathbf{b}^{\prime}$ share horoasymptotic directions.

Theorem 5.2 i) A generic surface $M \subset H_{+}^{4}(-1)$ all whose points are horohyperbolic has either two or four globally defined horoasymptotic fields that may eventually coincide pairwise over a closed subset of semiumbilic points.

ii) If $M$ is totally semiumbilical with isolated horospherical points and such that all its points are horohyperbolic, then $M$ has either one or two horoasymptotic fields globally defined that may eventually coincide over some closed subset.

Proof: i) In this case the horohyperbolicity ensures the existence of either two or four globally defined horobinormal fields which cannot be coincident at any point, for in this case it would be horoparabolic. Then Lemma 5.1 a) guarantees the existence of either two or four horoasymptotic fields respectively over the open submanifold $M_{3}$. Part ii) of Lemma 5.1 tells us that the horoasymptotic directions coincide by pairs over the semiumbilic points. ii) In this case the horohyperbolicity condition ensures the existence of either two or four horobinormals at each point. Due to the absence of horoparabolic points, we can assert that these determine globally defined horobinormal fields on $M$, which in turn determine either one or two horoasymptotic fields. Again, we have that the 
horobinormal pairs may be coincident at points for which the image of $A_{\mathbf{x}}$ is tangent to the cone $C$ and this determines that the two horoasymptotic directions will also be coincident at such points.

Corollary 5.3 A generic surface in $H_{+}^{4}(-1)$ exclusively made of horohyperbolic points which is compact without boundary and has nonvanishing Euler number has horospherical points.

Proof: This follows immediately by applying the Poincaré-Bendixon index formula to any of the horoasymptotic direction fields guaranteed by part i) of Proposition 5.2.

We observe that the same assertion is valid for surfaces satisfying either of the conditions ii) or iii) in Proposition 5.2.

It is worth to recall at this point the Carathéodory's conjecture. This asserts that a 2 -sphere immersed in $\mathbb{R}^{3}$ has at least two umbilic points. We observe that the inverse of stereographic projection takes umbilics of surfaces in 3-space to inflection points of their images in the $S^{3}$ considered as surfaces in euclidean 4-space. This naturally leads to the following generalized Carathéodory's conjecture: 2-spheres convexly embedded in $\mathbb{R}^{4}$ have at least two inflection points, where we observe that the convexity property is equivalent to the global existence of asymptotic directions (see [1] for a proof in the case of generic surfaces in euclidean 4-space). Now, by considering the analogy of this situation with the case treated here, we establish the following

Conjecture: $A$ 2-sphere immersed as an everywhere horohyperbolic surface in hyperbolic 4-space has at least two horospherical points.

We finally find a necessary condition for the semiumbilicity of a spacelike surface in terms of the horoasymptotic directions.

Theorem 5.4 Suppose that $M$ is a surface in $H_{+}^{4}(-1)$ with two globally defined horoasymptotic fields and isolated horospherical points. If $M$ is totally semiumbilical then the horoasymptotic directions are mutually orthogonal everywhere except at the horospherical points.

Proof: First of all we observe that $M$ is totally semiumbilical if and only there exist two normal fields $\nu_{1}, \nu_{2}$, locally defined and linearly independent at every non umbilical point of $M$, such that $M$ is $\nu_{i}$-umbilical ([5], Theorem 5.6). On the other hand, we have that $M$ admits two linearly independent normal fields locally defined for each $\mathrm{x} \in M$ for which $M$ is umbilical if and only if $M$ admits a unique principal configuration. In fact, given $\mathbf{x} \in M$, consider isothermal coordinates $\{u, v\}$ in a neighbourhood $U_{\mathbf{x}}$ of $\mathbf{x}$ and suppose that $\nu^{1}$ and $\nu^{2}$ are normal fields defined on $U_{\mathrm{x}}$ such that $M$ is $\nu^{j}$-umbilical. Without loss of generality we can take $\nu^{1}$ as the position vectorfield on $M$ and $\nu^{2}$ as a vectorfield 
$\nu$ tangent to $H_{+}^{4}(-1)$. We can take now another normal field $\xi$ such that $\{\rho, \xi, \nu\}$ defines a pseudo-orthonormal frame for the normal bundle $N U_{\mathbf{x}}$. Then given any normal field $\eta$, we can write $\eta=k_{1} \rho+k_{2} \xi+k_{3} \nu$, for appropriate smooth functions $k_{1}, k_{2}, k_{3}: U \rightarrow \mathbb{R}$. The coefficients of the second fundamental form in the direction of $\eta$ are given by

$$
\begin{aligned}
& e_{\eta}=\left\langle\frac{\partial^{2} \mathbf{X}}{\partial x^{2}}, k_{1} \rho+k_{2} \xi+k_{2} \nu\right\rangle=k_{1} e_{\rho}+k_{2} e_{\xi}+k_{3} e_{\nu}, \\
& f_{\eta}=\left\langle\frac{\partial^{2} \mathbf{X}}{\partial x \partial y}, k_{1} \rho+k_{2} \xi+k_{3} \nu\right\rangle=k_{1} f_{\rho}+k_{2} f_{\xi}+k_{3} f_{\nu}, \\
& g_{\eta}=\left\langle\frac{\partial^{2} \mathbf{X}}{\partial y^{2}}, k_{1} \rho+k_{2} \xi+k_{3} \nu\right\rangle=k_{1} g_{\rho}+k_{2} g_{\xi}+k_{3} g_{\nu} .
\end{aligned}
$$

And the equation of the curvature lines in this coordinates becomes (see [5], [11])

$h\left(f_{\xi} d u^{2}+\left(g_{\xi}-e_{\xi}\right) d u d v-f_{\xi} d v^{2}\right)+k_{1}\left(f_{\nu^{1}} d u^{2}+\left(g_{\nu^{1}}-e_{\nu^{1}}\right) d u d v-f_{\nu^{1}} d v^{2}\right)+$ $k_{2}\left(f_{\nu^{2}} d u^{2}+\left(g_{\nu^{2}}-e_{\nu^{2}}\right) d u d v-f_{\nu^{2}} d v^{2}\right)=0$.

Since $M$ is $\rho$ - and $\nu$-umbilical, we have that $e_{\rho}(\mathbf{x})=g_{\rho}(\mathbf{x}), f_{\rho}(\mathbf{x})=0$ and $e_{\nu}(\mathbf{x})=g_{\nu}(\mathbf{x}), f_{\nu}(\mathbf{x})=0$, for all $\mathbf{x} \in M$ and thus $f_{\rho} d u^{2}+\left(g_{\rho}-e_{\rho}\right) d u d v-f_{\rho} d v^{2}=$ 0 and $f_{\nu} d u^{2}+\left(g_{\nu}-e_{\nu}\right) d u d v-f_{\nu} d v^{2}=0$ Therefore, the principal configuration associated to $\eta$ is given by $h\left(f_{\xi} d u^{2}+\left(g_{\xi}-e_{\xi}\right) d u d v-f_{\xi} d v^{2}\right)=0$. So both fields $\eta$ and $\xi$ have the same principal configurations.

Conversely, given $M \subset H_{+}^{4}(-1)$, we know that it is $\rho$-umbilical, where $\rho$ is the position field. Take $\mathrm{x} \in M$ and let $\eta_{1}$ and $\eta_{2}$ be normal fields on a neighbourhood $U_{\mathbf{x}}$ of $\mathbf{x}$ in $M$, lying in $T_{\mathbf{x}} H_{+}^{4}(-1)$ (i.e., pseudo-orthogonal to $\rho$ ) that are linearly independent on $U_{\mathbf{x}}$. Their respective principal configurations are given by the equations $f_{\eta_{i}} d u^{2}+\left(g_{\eta_{i}}-e_{\eta_{i}}\right) d u d v-f_{\eta_{i}} d v^{2}=0$, for $i=1,2$. Since $M$ admits a unique principal configuration, we must have that $f_{\eta_{1}}=\lambda f_{\eta_{2}}$ and $g_{\eta_{1}}-e_{\eta_{1}}=\lambda\left(g_{\eta_{2}}-e_{\eta_{2}}\right)$, for some function $\lambda$ on $U_{\mathbf{x}}$. Taking $\bar{\nu}=\eta_{1}-\lambda \eta_{2}$ we have that $f_{\bar{\nu}}=f_{\eta_{1}}-\lambda f_{\eta_{2}}=0$ and $g_{\bar{\nu}}-e_{\bar{\nu}}=g_{\eta_{1}}-e_{\eta_{1}}-\lambda\left(g_{\eta_{2}}-e_{\eta_{2}}\right)=0$. Therefore $M$ is $\bar{\nu}$-umbilical. We observe that $\bar{\nu}$ and $\rho$ are linearly independent on $U_{\mathbf{x}}$, for they are pseudo-orthogonal.

Finally, suppose that $\theta_{1}$ and $\theta_{2}$ are the two distinct horoasymptotic fields globally defined on $M$ and let $b_{i}, i=1,2$ be the corresponding horobinormal fields, which must be distinct too. By taking appropriate coordinates on $M$ we can see that the direction $\theta_{i}$ is a principal direction for the shape operator $S_{b_{i}}$ and that its corresponding principal curvature vanishes everywhere. Since $M$ has a unique principal configuration, we have that the principal configurations of $b_{1}$ and $b_{2}$ coincide and thus $\theta_{2}$ (resp. $\theta_{1}$ ) must be the principal direction of $b_{1}$ (resp. $b_{2}$ ) corresponding to the nonvanishing principal curvature. But this means that $\theta_{1}$ and $\theta_{2}$ must be everywhere orthogonal, except at the critical points of the principal configurations.

Remark: In the case of a surface $M$ immersed in Euclidean 4-space, we have that the above is also a sufficient condition for total semiumbilicity ([12]). This is due to the fact that it can be shown that if $b_{i}, i=1,2$ are the horobinormal fields on $M$ and $k_{i}, i=1,2$ are the corresponding non vanishing curvatures, then the normal field $\nu=k_{2} b_{1}-k_{1} b_{2}$ is umbilical over $M$, and in this case this is a 
sufficient condition for semiumbilicity of $M$. In the horospherical case, we need to require the existence of some umbilical field over $M$ that is everywhere tangent to $H_{+}^{4}(-1)$, and to be able to ensure this we should have that $\nu=k_{2} b_{1}-k_{1} b_{2}$ is not a multiple of the position vectorfield $\rho$ over $M$. It is not clear at all that this needs to be true on any surface having everywhere orthogonal horoasymptotic fields. In any case we can assert that under the orthogonality assumption on the horoasymptotic fields $M$ is totally semiumbilical provided the normal field $\nu=k_{2} b_{1}-k_{1} b_{2}$ is not a multiple of $\rho$.

\section{References}

[1] R.A. Garcia, D.K.H. Mochida, M.C. Romero-Fuster and M.A.S. Ruas, Inflection Points and Topology of Surfaces in 4space, Transactions of AMS. 352 (2000), 3029-3043.

[2] M.Golubitsky and V.Guillemin,Stable Maps and Their Singularities, Grad. Texts in Maths., Springer-Verlag,New York (1973).

[3] S. Izumiya, D. Pei and T. Sano, Singularities of hyperbolic Gauss maps. To appear in Proceedings of the LMS. (2000).

[4] S. Izumiya, D. Pei and M.C. Romero Fuster, The lightcone Gauss map of a spacelike surface in Minkowski 4-space. Preprint (2001).

[5] S. Izumiya, D. Pei and M.C. Romero Fuster, Umbilicity of spacelike submanifolds of Minkowski space. Preprint (2002).

[6] J. Martinet, Singularities of Smooth Functions and Maps, London Math. Soc. Lecture Note Series, Cambridge Univ. Press, vol 58 (1982)

[7] D.K.H. Mochida, M.C. Romero-Fuster and M.A.S. Ruas, The geometry of surfaces in 4-space from a contact viewpoint. Geometriae Dedicata 54 (1995), 323-332.

[8] D. K. H. Mochida, M. C. Romero-Fuster and M. A. S. Ruas, Inflection points and nonsingular embeddings of surfaces in $\mathbb{R}^{5}$. To appear in Rocky Mountain Journal of Maths.

[9] J.A. Montaldi, On contact between submanifolds. Michigan Math. J. 33 (1986), 195-199.

[10] S.M. Moraes, M.C. Romero-Fuster and F. Sánchez-Bringas, Principal configurations and umbilicity of submanifolds in $\mathbb{R}^{N}$. Preprint (2001). 
[11] A. Ramirez-Galarza and F. Sánchez-Bringas, Lines of Curvature near Umbilical Points on Surfaces Immersed in $\mathbb{R}^{4}$. Annals of Global Analysis and Geometry 13 (1995), 129-140.

[12] M.C. Romero-Fuster and F. Sánchez-Bringas, Umbilicity of surfaces with orthogonal asymptotic lines in $\mathbb{R}^{4}$. Differential Geometry and Applications 16 (2002),213-224

Shyuichi Izumiya, Department of Mathematics, Hokkaido University, Sapporo 060-0810, Japan

e-mail:izumiya@math.sci.hokudai.ac.jp

Donghe Pei, Department of Mathematics, North East Normal University, Changchun 130024, P.R. China e-mail:northlcd@public.cc.jl.cn

María del Carmen Romero Fuster, Departament be Geometria i Topologia, Universitat be València, 46100 Burjassot (València), Espanya

e-mail: carmen.romero@post.uv.es 